\title{
Response to the letter by Sharma et al.: Intracutaneous sterile water injection versus oral paracetamol for renal colic during pregnancy: a randomized controlled trial. Int Urol Nephrol 2013; 45:321-325, DOI 10.1007/s11255-013-0405-3
}

\author{
Peng Xue
}

Received: 26 June 2013/Accepted: 8 July 2013/Published online: 12 March 2014

(C) Springer Science+Business Media Dordrecht 2014

1. We read this article with interest where the authors have compared oral paracetamol $1 \mathrm{~g}$ with intracutaneous sterile water injection at most painful site for renal colic in pregnancy. The authors showed that intracutaneous sterile water injection acts faster and significantly better in pain relief as compared to oral paracetamol. There are few points that need clarification. As mentioned in the manuscript, intracutaneous sterile water injection causes significant pain at the local site. The authors should have evaluated this pain at local site by visual analogue scale (VAS). Moreover, patient satisfaction with analgesic should have been reported.

Answer: Thank you for your kind letter and giving us constructive advises. We have used visual analogue scale (VAS) to evaluate the severity of pain in our manuscript. Meantime, to record the patient satisfaction is a good advice and required further studies. We did not show the patient satisfaction in our manuscript because our data were incomplete.

2. Another issue is that the authors compared intracutaneous sterile water injection with oral paracetamol which is a poor analgesic. The time required by paracetamol to achieve maximum plasma concentration is more than $1 \mathrm{~h}$. However, the authors have started rescue analgesia after 1 h. In our opinion, intramuscular paracetamol could have been used instead of oral paracetamol.

Answer: As we mentioned in our manuscript, we evaluated the anodyne and immediate effects of oral paracetamol as a control in this study, but we did not compare the effects of sterile water injections to those of intravenous paracetamol because (1) the safety of intravenous paracetamol has not been previously evaluated in the management of pain associated with renal colic in pregnant women, and (2) oral paracetamol is classified as grade B according to the US Federal Drug Administration pregnancy categories, a system of classifying drugs based on their established risks for use during pregnancy.

3. Another point that needs clarification is that the patients with ureteral stone complain of recurrent colic and therefore require repeated doses of analgesia. The site of injection if the patient needs multiple injections for the same condition is a matter of debate.

Answer: The initial management of symptomatic ureteric stones during pregnancy should be conservative, and most renal colic could be relieved with conservative therapy. However, when conservative approach fails, URSL may be a good alternative approach for definitive treatment [1].

\section{References}

1. Isen K, Hatipoglu NK, Dedeoglu S, Atilgan I, Caça FN, Hatipoglu $\mathrm{N}$ (2012) Experience with the diagnosis and management of symptomatic ureteric stones during pregnancy. Urology 79(3): 508-512
P. Xue $(\bowtie)$

Lianyungang, China

e-mail: xuepsun@126.com 